\title{
Highlights from the 60th national meeting of the Italian Society of Nephrology: celebrating the role of the nephrologist
}

\author{
Luca De Nicola ${ }^{1}$ Filippo Aucella ${ }^{2} \cdot$ Giuliano Brunori $^{3}$
}

Published online: 3 September 2020

(C) Italian Society of Nephrology 2020

"I was led by hand to choose a job that is the only one to me, the only one that allows me achieving myself in the most joyful, most immediate, form".

This statement by Federico Fellini, the most famous Italian filmmaker (Fig. 1), was the incipit of the last national meeting of the Italian Society of Nephrology (SIN), held 2-5 October, 2019 in Rimini, Fellini's hometown. The claim certainly represents the spirit of the meeting. The scientific committee, in fact, wanted to celebrate the 60th anniversary of our Society by placing emphasis on the practice of nephrology.

Nephrologists can be efficacious in improving the prognosis of their patients; however, this task requires passion, curiosity, and flexibility. In particular, updating one's own knowledge of nephrology on the basis of new scientific discoveries is a relevant aspect. Indeed, since its birth in the 50 's, the field of nephrology has made major progress with regard to renal replacement therapy (RRT) and conservative therapies. Treatment of the complete loss of kidney function has improved thanks to the progress of technological (dialysis) and pharmacological (epoietin and agents for CKD-mineral bone disease above all) tools and because of improved immunosuppression for kidney transplant recipients. Improvements in therapy significantly ameliorated the prognosis of RRT patients. More innovation in dialysis is

Electronic supplementary material The online version of this article (https://doi.org/10.1007/s40620-020-00856-4) contains supplementary material, which is available to authorized users.

Luca De Nicola

luca.denicola@unicampania.it

$1 \quad$ Nephrology and Dialysis Unit, Department Advanced Medical and Surgical Sciences, University of Vanvitelli, Naples, Italy

2 Nephrology and Dialysis Unit, IRCCS "Casa Sollievo Della Sofferenza" Scientific Institute for Research and Health Care, San Giovanni Rotondo, Italy

3 Nephrology and Dialysis Unit, Hospital of Trento, Trento, Italy expected; efforts will soon be dedicated to improving vascular access function, biocompatibility of materials, and treatment safety [1].

Over the last few decades, nephrologists have also observed a substantial attenuation of CKD progression to a rate close to the physiological age-related decline of glomerular filtration rate (GFR). Indeed, collected data regarding 729 CKD patients who were regularly followed-up in an Italian outpatient renal clinic (mean age 64 years; males 59\%; diabetes $35 \%$; cardiovascular disease $44.9 \%$, estimated glomerular filtration rate [eGFR] $48 \mathrm{~mL} / \mathrm{min} / 1.73 \mathrm{~m}^{2}$, proteinuria $0.9 \mathrm{~g} / 24 \mathrm{~h}$ ) revealed an overall decline in eGFR averaging $0.99 \mathrm{~mL} / \mathrm{min} /$ year (Table 1) [2]. Again, this improvement in the renal prognosis of non-dialysis chronic kidney disease (CKD) patients mainly derives from the discovery and implementation of effective treatments [nutritional intervention, inhibition of the renin-angiotensin-aldosterone system(RAAS), above all], and the increased awareness among non-nephrologists of the importance of nephrology follow-up as well.

The nephrology community is therefore coping with a revolution in the conservative treatment of $\mathrm{CKD}$ that has shifted from "the point of no return", that is, the assumption that the protective effects of any intervention in CKD become useless when serum creatinine reaches $1.5-2.0 \mathrm{mg} /$ $\mathrm{dL}$ [3], to the new concept that regular nephrology care can indeed postpone the need for RRT for many years, even in patients with severe CKD [4].

In Italy, the PIRP project (Prevenzione Insufficienza Renale Progressiva) has provided real-life evidence of the importance of timely nephrology referral [5]. This project is currently evaluating the long-term effects of established collaboration between general practitioners and nephrologists in more than 25,000 CKD patients residing in Emilia-Romagna Region. The first and most outstanding finding is the $10 \%$ decrease between 2006 and 2016 in the incidence of patients starting dialysis in the Region (which was closely linked to the $68 \%$ reduction observed within the PIRP cohort. 


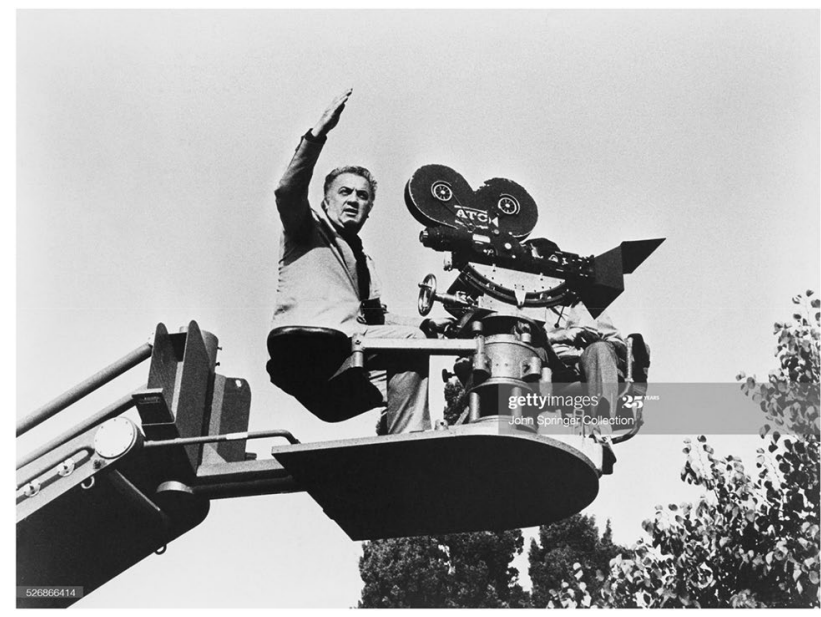

Fig. 1 Federico Fellini, Italian filmmaker. Rimini 1920-Rome 1993

Table 1 CKD progression, overall and by primary kidney disease, estimated in 729 pts with CKD stage 1-4 after the first year of nephrology care (six eGFR values over a median follow up of 3.3 years, interquartile range 1.9-5.1) [2]

\begin{tabular}{lll}
\hline Patients & $\begin{array}{l}\text { eGFR decline } \\
(\mathrm{mL} / \mathrm{min} / \mathrm{year})\end{array}$ & Interquartile range \\
\hline All & 0.99 & -3.33 to +1.14 \\
Hypertensive Nephropathy & 0.62 & -2.70 to +1.30 \\
Tubulointerstitial Nephropathy & 0.77 & -3.09 to +1.42 \\
Glomerulonephritis & 1.34 & -3.85 to +1.33 \\
Diabetic Kidney Disease & $1.39^{*}$ & -3.73 to +0.43 \\
Polycystic Kidney Disease & $3.70^{\#}$ & -5.16 to -1.61 \\
\hline
\end{tabular}

$* \mathrm{P}=0.016$ and ${ }^{\#} \mathrm{P}=0.001$ vs reference (Hypertensive Nephropathy)

Noteworthy, the role of nephrology, both with regard to clinical practice and as a research area, will soon become even more pivotal when considering the epidemic of CKD. Kidney diseases are in fact an unquestionable global public priority with a world population of patients now exceeding 850 million, which is actually twice the number of diabetic individuals and more than 20 times higher than the patient population with AIDS/HIV [6]. More importantly, 1.2 million deaths were primarily ascribed to CKD in 2017, with a $42 \%$ increase as compared to 1990 , and this estimate is projected to increase two- to three-fold by 2040 when CKD is expected to climb the ranking of the causes of years of life lost (YLL) from the current 16th place to 5th place [7].

While this epidemiological picture on a world-wide level is clearly worrisome, strategies to effectively limit the growth and severity of kidney diseases are being developed. On July 10, 2019 the US President signed the Executive Order on Advancing American Kidney Health [1], a multifaceted "declaration of war" against CKD with predefined and ambitious objectives including a $25 \%$ reduction of end stage kidney disease (ESKD) patients by 2030, an increase of up to $80 \%$ of the new ESKD patients being treated at home (either with peritoneal or extracorporeal dialysis) by 2025, and doubling the access to kidney transplant by 2030 , especially by reinforcing programs for living donors. The latter point takes on critical relevance when considering the survival advantage of patients receiving a kidney from a living donor as compared to a deceased donor and the importance especially for dialysis patients. In 2009 , after having collecting data from a large sample of 7948 patients with 35,515 observation years, the Dutch National Organ Transplant Registry demonstrated that 10 -year survival was on average higher in the preemptive living-donor kidney transplantation setting (73\%) as compared to the program of deceased-donor kidney transplantation (45\%), with the two options being, as expected, remarkably different versus patients treated with dialysis (11\%) [8].

The importance of the prevention of ESKD, as well as of home replacement therapies has further increased recently due to the COVID-19 pandemic. The SIN rapidly distributed a survey among all the Italian nephrology and dialysis units which was met with a high response rate (365 centres, i.e. $80.4 \%$ of all centres in Italy) [9]. Of note, to date, this remains the only national survey conducted on COVID-19 in the RRT population. Results are highly informative. Infection risk during the COVID-19 outbreak was found to be substantially higher -more than 10 times greater- in ESKD as compared to the general population, with a risk about 3 -fold lower in RRT patients treated at home. The incidence rate of positive cases was $3.6 \%$ for in-centre hemodialysis (HD) versus $1.4 \%$ for peritoneal dialysis (PD) and $0.9 \%$ for transplanted patients (RTX). On the other hand, one infected patient out of three died regardless of RRT modality, thus with a mortality rate of COVID-19 that was about 2.5-fold higher as compared to the general population.

The "war" that has nephrologists on the frontlines can be extended to other multidisciplinary areas of intervention that are in continuous evolution, namely diabetes, geriatric syndromes and cancer. In all these settings it is reasonable to assume that a nephrologist would be needed to help improve research, guidelines and clinical practice. From this point of view, it is worth highlighting that the SIN has recently started collaborating with other scientific societies to face these new tasks.

Under this new perspective of nephrology, we collected seven papers from prominent speakers at the 60th national meeting of our Society in order to convert their lectures into "hard" documents on the present and future of nephrology management. These selected reviews will hopefully constitute the starting point for the future scientific and clinical growth of nephrology. Of course, limited availability of space and speakers prevented us from covering the whole spectrum of renal diseases. Nonetheless, 
further studies generated by the new era of nephrology are expected and papers will be welcome in the Journal of Nephrology that has now become a first-rate Journal in the field of nephrology.

The first day of the Meeting was entirely dedicated to the multidisciplinary approach to diabetic kidney disease (DKD). Nowadays, DKD represents the main cause of ESKD in the western world; it affects about 4 diabetics out of 10 with dramatic consequences on their prognosis. Two speakers addressed the unmet needs of these highrisk patients and provided possible solutions. Federica Barutta, from the Laboratory of Diabetic Nephropathy at the University of Turin, describes the most recent findings of experimental studies with novel therapeutic targets, and discusses how translation of preclinical research to humans might be improved. In line with the research on DKD, Dick de Zeeuw, from the University of Groningen in the Netherlands -the "cradle" of major trials in nephrology-, provides a critical reappraisal of the current clinical research and anticipates the up-coming changes in trial design aimed at favouring the timely implementation of derived evidence into clinical practice.

Among the causes of CKD, both DKD and Polycystic Kidney Disease definitely remain the most progressive renal diseases (Table 1); however, the high risk of precipitating to the dialytic stage is often independent of the primary diagnosis. Observational studies in the setting of renal clinics provide estimates of ESKD incidence rates ranging between 4 and 8 events per 100 patientyears, mainly related to basal renal function (Figure S1). A prominent determinant of ESKD onset is represented by intercurrent acute kidney injury (AKI) episodes that can de facto nullify all the therapeutic efforts made by nephrologists. This critical issue is addressed by Johanna Kurzhagen from the research group at the Johns Hopkins University School of Medicine-Baltimore headed by Hamid Rabb that for many years has been devoted to the study of AKI pathophysiology. Authors provide an overview of the mechanisms underlying AKI-related kidney fibrosis and discuss research gaps and novel therapeutic interventions.

As mentioned, a new actor now coming into the already complex scenario of AKI and CKD is cancer with its "good fellas" of underlying comorbidities and treatment regimens that are often associated with nephrotoxic effects. Noteworthy, besides and beyond the creation of a multidisciplinary working group by the SIN, Italy recently hosted an international conference specifically aimed at building the basis of Onco-Nephrology. Laura Cosmai, an Italian nephrologist with consolidated experience in this field, adds further knowledge by answering practical questions: When should a nephrologist be involved in the management of cancer patients? Which patients should be referred for onconephrological evaluation?
Which conditions should be treated? How should an OncoNephology clinic be created?

In the clinical history of CKD patients, dialysis is the natural outcome. This occurs more frequently in patients who are followed-up in tertiary nephrology care (Figure S1), while unreferred subjects more often die before reaching the dialytic stage. Of great interest, CKD patients receiving regular pharmacological and non-pharmacological treatment prior to ESKD also survive longer than unreferred individuals during the subsequent dialysis period, thus supporting the idea that the quality of management in non-dialysis stages of CKD mainly dictates the prognosis of patients starting a dialysis program. The latter finding, together with the observed decrease in the rate of GFR decline in the nondialysis stage of CKD, should allow us to draw a more encouraging picture on the current epidemiology of the dialysis population. This hypothesis is supported by the current report of the Italian Dialysis and Transplantation Registry, prepared by Maurizio Nordio, Aurelio Limido and Maurizio Postorino, the reference panel of the Italian Registry for several years, which testifies to the stabilization of the incidence of treated ESKD over the last years and a more favourable outcome of RRT patients in Italy versus other countries.

Although signals of better prognosis of treated ESKD are evident nowadays, RRT can further improve the picture. Sydney Tang, Chairman of the Hong Kong Society of Nephrology, describes current evidence supporting the socalled "PD-first" policy to promote PD as the ideal form of dialysis prior to kidney transplantation, and suggests ways to increase and support the utilization of PD. The content of this paper is of great clinical interest because it is drawn from direct experience; Hong Kong has in fact the highest PD utilization rate in the world, being used in $75-80 \%$ of all dialysis patients. Of particular interest is the use of incremental PD as the first-choice RRT; this strategy is based on the elective start of low-dose PD that is gradually uptitrated to maintain homeostasis adequacy in parallel with the progressive loss of residual renal function. This "soft and tailored" modality of dialysis, which is well known to Italian nephrologists, is safe and allows delaying full-dose dialysis by about 12 months.

"Last but not least" is the topic addressed by Simona Granata from the excellent research group headed by Gambaro and Zaza at the University of Verona. Authors discuss pathophysiology and potential novel treatment of kidney allograft fibrosis, which is a major research target in nephrology at large. Interestingly, in fact, innovative antifibrotic agents which were originally created to prolong graft survival may also play a role in the pharmacological therapy of nondialysis CKD to slow down progression to ESKD.

Overall, the papers selected for the SIN 2019 Highlights provide evidence on how nephrology has changed and how 
it will change in the future. Nephrologists should read these papers to improve their awareness of the impressive progress that has been made by the speciality and, therefore, to become more confident with the refrain of the 2019 National Meeting of SIN: "Yes we can, Yes we care!".

\section{Compliance with ethical standards}

Conflict of interest Authors declare no conflict of interest.

Ethical approval This article does not contain any studies with human participants or animals performed by any of the authors.

\section{References}

1. Rosenberg ME, Ibrahim T (2019) Winning the war on kidney disease: perspective from the American Society of Nephrology. Clin J Am Soc Nephrol 14(12):1792-1794

2. De Nicola L, Provenzano M, Chiodini P, Borrelli S, Garofalo C, Pacilio M, Liberti ME, Sagliocca A, Conte G, Minutolo R (2015) Independent role of underlying kidney disease on renal prognosis of patients with chronic kidney disease under nephrology care. PLoS One 10(5):e0127071

3. Maschio G, Oldrizzi L, Rugiu C (1991) Is there a "point of no return" in progressive renal disease? J Am Soc Nephrol 2(4):832-840

4. Pacilio M, Minutolo R, Garofalo C, Liberti ME, Conte G, De Nicola L (2016) Stage 5-CKD under nephrology care: to dialyze or not to dialyze, that is the question. J Nephrol 29(2):153-161

5. Santoro A, Gibertoni D, Rucci P, Mancini E, Bonucchi D, Buscaroli A, Campagna A, Cappelli G, David S, Gregorini MC, La
Manna G, Mosconi G, Rigotti A, Scarpioni R, Storari A, Mandreoli M (2019) The PIRP project (Prevenzione Insufficienza Renale Progressiva): how to integrate hospital and community maintenance treatment for chronic kidney disease. J Nephrol 32(3):417-427

6. Jager KJ, Kovesdy C, Langham R, Rosenberg M, Jha V, Zoccali C (2019) A single number for advocacy and communication-worldwide more than 850 million individuals have kidney diseases. Kidney Int 96(5):1048-1050

7. Foreman KJ, Marquez N, Dolgert A, Fukutaki K, Fullman N, McGaughey M, Pletcher MA, Smith AE, Tang K, Yuan CW, Brown JC, Friedman J, He J, Heuton KR, Holmberg M, Patel DJ, Reidy P, Carter A, Cercy K, Chapin A, Douwes-Schultz D, Frank T, Goettsch F, Liu PY, Nandakumar V, Reitsma MB, Reuter V, Sadat N, Sorensen RJD, Srinivasan V, Updike RL, York H, Lopez AD, Lozano R, Lim SS, Mokdad AH, Vollset SE, Murray CJL (2018) Forecasting life expectancy, years of life lost, and all-cause and cause-specific mortality for 250 causes of death: reference and alternative scenarios for 2016-40 for 195 countries and territories. Lancet 392(10159):2052-2090

8. Liem Y, Weimar W (2009) Early living-donor kidney transplantation: a review of the associated survival benefit. Transplantation 87(3):317-318

9. Quintaliani G, Reboldi G, Di Napoli A, Nordio M, Limido A, Aucella F, Messa P, Brunori G, Italian Society of Nephrology COVID-19 Research Group (2020) Exposure to novel coronavirus in patients on renal replacement therapy during the exponential phase of COVID-19 pandemic: survey of the Italian Society of Nephrology. J Nephrol. https://doi.org/10.1007/s40620-020-00794 -1 [published online ahead of print, $2020 \mathrm{Jul} \mathrm{3}$ ]

Publisher's Note Springer Nature remains neutral with regard to jurisdictional claims in published maps and institutional affiliations. 